\title{
RISK REDUCTION IN TRANSPORT SYSTEM IN EMERGENCY CONDITIONS: A FRAMEWORK FOR SUPPLY ANALYSIS
}

\author{
GIUSEPPE MUSOLINO \\ DIIES - Dipartimento di Ingegneria dell'Informazione, delle Infrastrutture e dell'Energia Sostenibile \\ Università Mediterranea di Reggio Calabria, Italy
}

\begin{abstract}
There is a rich literature, mainly developed in the last two decades, about risk reduction in transport system in emergency conditions. One of the challenges regards the development of ad-hoc transport system models (TSMs) in order to simulate emergency conditions. The paper focuses on the supply (network) component of the TSMs. The general objective of the research is the development of a framework capable to capture the relevant changes of the network from the ordinary conditions, due to an approaching calamitous event. The research will consider non-stationary conditions in transport supply models for continuous services. In relation to the consolidated static models, this evolution is necessary to capture the dynamics of the transportation network during the evacuation in terms of both topology (e.g. link closure, contraflow) and capacity of the links and of the network. The concept of learning process will be introduced to take into account how the transport costs (disutilities) in ordinary and emergency conditions will be perceived by the users.
\end{abstract}

Keywords: risk, transport system models, supply, evacuation, learning process.

\section{INTRODUCTION}

Risk is commonly defined as the product of three probabilities: occurrence, vulnerability, exposure. Transport system planning aims to reduce exposure, by means of evacuation procedures. The objectives of urban evacuations concern the minimization of the number of people and the quantity of goods involved in a calamitous event [1].

A fundamental element to support the evacuations planning and optimization is the transport system model (TSM) framework. TSMs in ordinary conditions are consolidated in the scientific literature. They are generally used by planners and technicians to identify the critical elements of the transport systems and to ex-ante assess alternative scenarios, or alternative transport system configurations, concerning both the demand (e.g. recurrent and no-recurrent demand peaks exceeding capacity), and the supply elements (e.g. new transport infrastructures or changes of existing services) [2]-[4].

However, TSMs in emergency conditions and/or when exogenous calamitous events affecting the transport system occur, have received minor attention in the literature. When an exogenous calamitous event starts its effects, both the demand and the supply components of the transportation system change in a relevant way. As far as concern demand, transport users travel on the network with different a behaviour (e.g. in terms of destination, mode, path choices), and they are not aware of network congestion, nor of reliability, of the transportation network. As far as concerns the supply, network in terms of both topology (e.g. link closure, contraflow) and capacity of the links and nodes changes, too.

Models and algorithms specified and calibrated in ordinary conditions for simulation and design transportation system cannot be directly applied in emergency conditions, when unexpected temporary over-saturation of some road links and nodes, queue formation and dispersion occur, and transport users are not aware of the current conditions of the network. 
In order to capture and simulate the relevant changes that occur in the transport systems in emergency conditions, it is necessary to adapt TSMs developed for ordinary conditions. It is necessary to take into account two main elements: the introduction of dynamics in models and procedures and the consideration of the influence of emerging ICTs, both on the supply and on the demand modelling components.

The paper deals with the research area connected to transport supply modelling in the context of the TSM framework. The research novelty of the paper concerns the introduction of elements that allow to capture the dynamics of the transportation network in emerging conditions (e.g. during the evacuation), in relation to the consolidated static supply models. In particular, the paper focuses on the concept of learning process (model), that explain (simulates) how transport users (evacuees) forecast the costs (or disutilities) of the transport network that they will experience during evacuation, from previous experience of the network and/or from existing information systems (e.g. emerging ICTs). The learning process could take into account different types of information provided by emerging ICTs (e.g. internet of things, big data), to update within-day and day-to-day information.

The remaining part of the paper is articulated as follows. Section 2 deals with the current research areas connected to evacuation planning and optimization. Section 3 presents the main characteristics of the of TSMs, focusing on the supply-demand interaction (traffic assignment) models and of supply models. Section 4 introduces the elements concerning the dynamic extension of supply model consistently with within-day and day-to-day information, provided by emerging ICTs. The final section reports the research perspectives.

\section{EVACUATION PLANNING AND OPTIMIZATION}

The topic of evacuation planning and optimization has received little attention in the literature. Some studies were produced in the early 2000s [1], [5]-[7]. After the 9/11 and Katrina, the study of emergency conditions received great impulse (see, as example, [8], and the references included); however, according to the author, the systematic development of research on evacuation planning and optimizations, with the actualization to the new frontiers given by emergent ICTs is not present the literature.

In general, the following five research areas may be considered.

- Emergency planning, which is part of the broader disaster management cycle that, at international level, is identified with the following keywords: prevention; mitigation or prevention; preparedness; response; recovery [9]-[11].

- Travel demand, where the user evacuation is the main activity inside the exposure regarding risk reduction in transport systems. The evacuation problem in the TSM approach needs the development of different components; two of them are considered: the demand analysis considering also stated preference surveys and the planning process considering parametric and non-parametric methods [12]-[14].

- Transport supply, where the objective is the development of ad-hoc models capable to capture the relevant changes of the network in emergency conditions, due to an approaching calamitous event [15], [16].

- Decision Support Systems (DSS), where it is necessary to consider appropriate DSS taking into account multimodality for the evacuation design. It is also necessary to build TSMs able to simulate non stationary conditions and considering risk, taking into account both the availability of reliable paths and peculiarity of path choice in emergency conditions [17]-[19]. 
- Network Design, where it is necessary to build TSMs concerning: the calibration of the cost functions, the design of the traffic junction regulation, the design of the routes for emergency vehicles [20]-[22].

\section{TRANSPORT SYSTEM MODEL (TSM)}

TSMs are able to represent each single component of the transport system and the mutual interactions.

The main three modelling components of TSMs are the traffic assignment, the transport supply and the travel demand. The core of the TSMs is the transport network-demand interaction (traffic assignment) model, which simulates how travel demand and transport networks interact in transportation systems. The two modelling components are: the transport supply (network) model, which simulates performances and traffic flows, resulting from users and from transport infrastructures and services; and the travel demand model, which simulate users' choices resulting from activities and infrastructure and service performances.

The following parts of this section present a classification of supply-demand interaction (traffic assignment) models (Section 3.1) and of supply models (Section 3.2), according to the scheme depicted in Fig. 1. Travel demand models are described in Russo and Chilà [14].

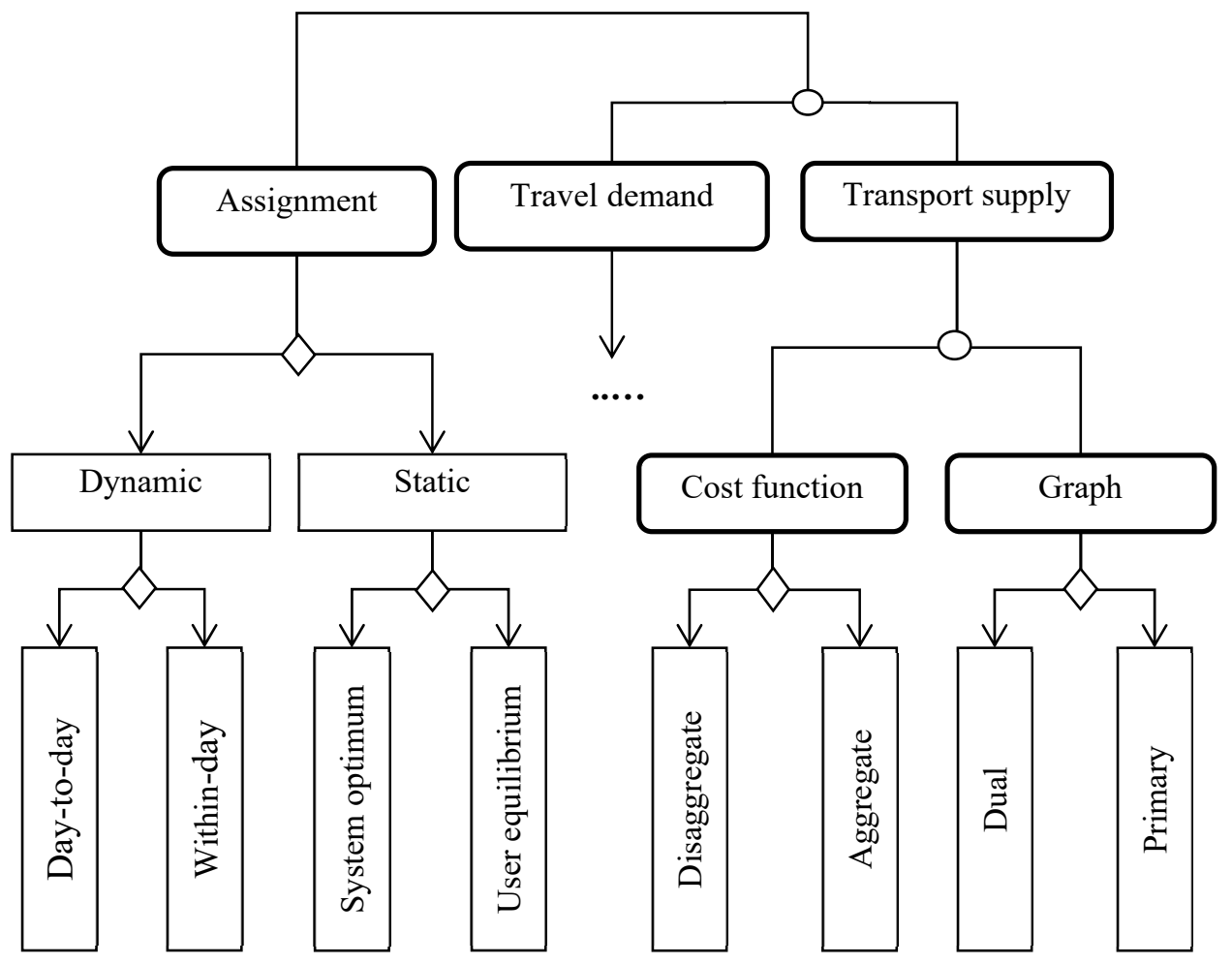

Legend

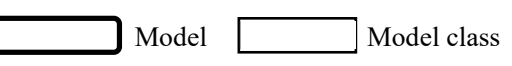

Stands for AND: the two models are parts of

Stands for OR: the model belongs to alternative classes

Figure 1: Classification of supply and of supply-demand interaction models. 


\subsection{Supply-demand interaction (traffic assignment) model}

Traffic Assignment (TA) models allow to calculate performances (costs) and vehicular flows on each element (link and nodes) of the transport network, resulting from origin-destination demand flows, users' path choice behaviour and link costs.

Supply-demand interaction models may be classified according to different criteria: path choice, static vs. dynamic, equilibrium vs. process approach.

As far as concerns path choice, the specification of path choice models is based on the definition of the path choice set and the path choice models. The path choice set is behaviourally defined, by selecting admissible paths according to behavioural rules of the user. Paths belonging to the path choice set can be variable or fixed, if new paths can be periodically reconsidered from the users or not.

Path choice models can be subdivided into two categories: pre-trip, if the user chooses the path according to the origin, the destination and other characteristics, before starting the trip; en-route, if the users modifies, during the trip, the path according to the current traffic conditions on the network.

Two types of path choice models can be identified: deterministic, if the users chooses the path of minimum cost (or disutility); stochastic, if the user associates a probability to each path belonging to the path choice set.

The en-route category of path choice models requires that information concerning the current and future traffic conditions of the network are available in some elements, such as diversion nodes. These information may be provided today by means of emerging ICTs, as briefly described below.

As far as concerns equilibrium vs. process approach, equilibrium is a (mathematical) condition, according to which path (link) flows are mutually consistent with the corresponding path (link) costs. This problem can be formulated with the so-called fixedpoint models, variational inequalities or optimization models [3], [4]. Equilibrium traffic assignment models of the literature are based on two approaches:

- User Equilibrium (UE), where path flows are estimated according to the First Wardrop principle [23], in order to minimize individual cost of users ;

- System Optimum (SO), where path flow-path cost relationship is consistent with the Second Wardrop principle [23]; in order to achieve the minimum total cost on the network, although some users may follow a non-minimum cost path.

The process approach is associated with non-equilibrium, generated by the independence of link cost variables on vehicular flows in the static case. Fixed costs or uncongested networks result in non-equilibrium traffic assignment models, while variable costs or congested networks lead to equilibrium traffic assignment models.

As far as concerns static vs. dynamic approach, TA models may be classified into:

- Static (STA) models, when travel demand, path choices and the transport network are consistent in a reference time (stationary conditions);

- Dynamic (DTA) models, when stationary conditions are removed and transport system evolution is represented in a reference time, as in the case of emergency conditions.

Therefore, STA models achieve reasonable results when the time horizon simulation is long enough to assume stationary conditions. On the contrary, DTA models are necessary when frequent changes in network and demand occur, like in emergency conditions, and the stationary hypothesis cannot be assumed. 
STA models belong to equilibrium category when the transport network is congested, in other words when link travel times depend on link flows (e.g. urban rods); while they belong to process category, such as network loading (NL) models, if link travel times do not depend on link flows (e.g. extra-urban roads). DTA models are generally associated to process category, even if the concept of dynamic equilibrium is available in the literature [24]. As matter of fact, it is reasonable to assume that users' choices in ordinary conditions may be based upon their experience of the network: when users have a certain experience of the network, equilibrium could be achieved and all users experience the same travel time. Emergency conditions are rare and, therefore, users have no experience of the network in these conditions. Hence, equilibrium, as defined in ordinary conditions, cannot be achieved.

DTA models may belong to two categories, that simulate two different temporal evolutions of the transport system [3], [4], [24], for an exhaustive description of the theoretical background):

- within-day DTA models, that simulate the within-day evolution of the transport system, that occurs over continuous time inside a given day; or inside a part of the day (e.g. morning peak hour);

- day-to-day DTA models, that simulate the day-to-day evolution of the transport system, that occurs over discrete time from (a part of) a given day and the next one.

\subsection{Transport supply (network) model}

According to the most common developed approach, transport supply models are represented by a network model. The network is composed by means of a graph, with links and nodes, and cost functions.

The graph can be primary versus dual. The cost functions can be classified according to different criteria: level of aggregation, element of the network.

According to the level of aggregation, cost functions range from a macroscopic to microscopic approach.

The macroscopic approach uses continuous flow representation and cost functions with aggregate variables (speed, density, flow) and with explicit capacity such as in static models. The representation of space and time can be continuous or discrete.

The microscopic approach is based on discrete flow representation allowing simulation of the behaviour of a single user. Cost functions are disaggregated in which the traffic flow is the result of individual choices, interactions among users and interactions between users and road facility.

An intermediate case is the mesoscopic approach where the behaviour of groups of users, termed packages, is simulated by means of a discrete flow representation [17], [18].

According to the element of the network, cost functions belong to two different classes:

- link, that simulate the (average) costs of users travelling on each link of the network;

- node, that simulate (average) costs of users at each node of the network (e.g. signalized and not-signalized intersections, merging and diverging areas, toll plazas).

Link models can be divided in two categories: macroscopic a microscopic.

Macroscopic link models are, generally, aggregate speed-density versus time-flow relationships, that estimate average values of traffic variables, as flow, density, speed.

Microscopic link models belong to two categories: car-following models, that simulate the movement of each vehicle and the interaction with vehicles travelling on the same lane; 
lane-changing models, that simulate the interactions among vehicles travelling on different lanes.

Among node models, there are models for signalized nodes and models for not-signalized nodes [21]. Models for signalized nodes can be subdivided in: macroscopic, for an aggregate analysis of node performances and microscopic, for a disaggregate analysis of node performances, simulating explicitly every driver behaviour. Models for not-signalized nodes are based on the gap-acceptance model. Different gap-acceptance models have been developed, according to the assumptions of the critical gap distribution.

A general static formulation of a congested supply model consists of two equations:

- path costs versus link costs consistency equation

$$
\mathbf{g}=\Delta^{\mathrm{T}} \boldsymbol{c}(\mathbf{f})
$$

- path flows versus link flows consistency equation:

$$
\mathbf{f}=\Delta \mathbf{h}
$$

where

- $\quad$ g, path costs vector;

- $\Delta$, link-path incidence matrix;

- $c()$, link cost functions vector;

- $\quad$ f, link flows vector;

- $\quad \mathbf{h}$, path flows vector.

The mutual relationships among the input/output variables of eqns (1) and (2) are reported in Fig. 2.

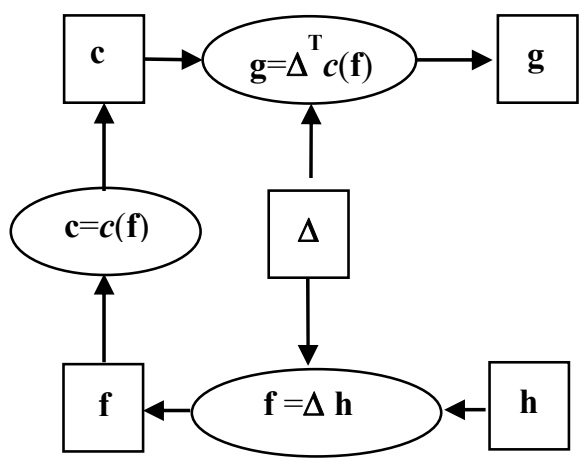

Figure 2: Mutual relationships among variable in a static supply model.

\section{LEARNING PROCESS IN PRESENCE OF EMERGING ICT}

The specification of a (dynamic) supply model consistent with a DTA model (see Section 3.1 ), according both to a within-day and a day-to-day pattern, requires an extension of the static supply model presented in eqns (1) and (2). In particular, it is necessary to include a modelling components related to the learning process of transport user, that could take into account also the different types of information (e.g. historical, real-time) provided by emerging ICT. Some elements are presented in the present section. 


\subsection{Emerging ICT}

New, or emerging, forms of Information and Communication Technologies (ICTs) have been developed in the last decade. They quickly spread in many areas of economy and society (e.g. manufacturing, social media, etc.), influencing the behaviour of people at individual and collective way. Some applications are proposed in the field of city logistics [25] and of ports and maritime transport [26], [27].

In broad terms, the different emerging technologies present in the market may be classified into four groups (see [25]-[28]):

- BlockChain (BC), or "distributed ledger" is a "technology for keeping a chronologically ordered list of transactions (a ledger) on multiple, independent stakeholders (called nodes). Updates of the list should be identical on each of the nodes and each node checks the validity of the transactions before updating its list" [27];

- Internet of Things (IoT) is a "framework that leverages on the availability of heterogeneous devices and interconnection solutions, as well as augmented physical objects providing a shared information base on global scale, to support the design of applications involving at the same virtual level both people and representations of objects" [28];

- Artificial Intelligence and Machine Learning (AI\&ML) aim to improving the prediction and decision making on transport operations, as well as to automatic repetitive (though sensitive) manual transactions;

- Big Data (BD) enrich information deriving from traditional data, in terms of volume and variety. ICT collects, manages and processes big data within a tolerable elapsed time, in order to improve the representation of traffic phenomena, and their modelling [25].

The $5 \mathrm{G}$ (or $6 \mathrm{G}$ ) communication network is not considered because it is transversal to all other technologies.

The above classes of emerging ICTs are able to provide new opportunities to define and implement integrated and dynamic solutions to optimize the different components of the TSM.

It is worth noting that these emerging ICTs are mature today and they are, generally, part of the stand-alone digital platform of almost every transport user and/or decision-maker (e.g. transport system manager). They may be considered emerging, as their implementation inside the Intelligent Transport Systems (ITSs) is still in fieri.

\subsection{Learning process}

The modification of users' knowledge about the network may be described by means of the learning process, that explain how transport users (e.g. evacuees) forecast today the costs (or disutilities) of the transport network (e.g. during an evacuation), from previous experience of the network (e.g. in ordinary conditions) and/or from previous forecasts (e.g. by means of emerging ICTs).

In broad terms, the learning process of individual users may be simulated with the (dis) utility updating model, that allows to obtaining the forecasted/computed costs in a period t.

In the case of path costs, the vector of forecasted (or computed) path costs at time $t, \mathbf{g}^{\mathbf{f o}, \mathrm{t}}$, may be expressed as follows: 


$$
\mathbf{g}^{\mathbf{f o}, \mathbf{t}}=g\left(\boldsymbol{\beta}, \mathbf{g}^{\mathbf{e x}, \mathbf{t}-\mathbf{1}}, \mathbf{g}^{\mathbf{f o}, \mathrm{t}-\mathbf{1}}\right)
$$

where

- $\quad g()$, implicit updating function that may be differently specified;

- $\quad \beta$, vector of parameters to be calibrated;

- $\quad \mathbf{g}^{\text {ex,t-1}}$, vector of experienced path costs at time $\mathrm{t}^{-1}, \mathbf{g}^{\mathbf{e x , t}-\mathbf{1}}$;

- $\mathbf{g}^{\mathbf{f o , t}-1}$, vector of forecasted (or computed) path costs at time $\mathrm{t}^{-1}, \mathbf{g}^{\mathbf{f o , t}-\mathbf{1}}$.

The implicit updating function, $g($, may be specified in different ways [4]. The simplest specification is the "yesterday" filter, where the forecasted path costs at time t are assumed to be equal only to the experienced path costs at time $\mathrm{t}^{-1}$ (yesterday). An extension of yesterday filter is the moving average filter, where the forecasted path costs at time $t$ depend on the experienced path costs at time $\mathrm{t}^{-1}, \mathrm{t}^{-2}, \mathrm{t}^{-3}, \ldots$ In the exponential filter specification, they are defined by means of a strict convex combination of forecasted and experienced costs at time $\mathrm{t}^{-1}$.

The learning process may evolve over time both according a within-day and day-to-day pattern. According to the literature, the within-day evolution is generally considered in continuous time, while the day-to-day evolution is generally considered in discrete time.

The new opportunities offered by emerging ICTs in providing historical, real-time and forecasted information can be exploited by introducing some modifications in the learning process, as traditionally specified in the classical problems.

Among the emerging ICTs classified in the previous paragraph, IoT, $B D$ and $A I$ may be considered the ones with the greatest impact on path choice [25]. According to their features, IoT allows real-time communication between vehicles (vehicle-to-vehicle communication) and between vehicles and infrastructure (vehicle-to-infrastructure communications), BD are able to provide an exhaustive and detailed representation of historical patterns of network costs, $A I$ could offer the possibility to support short-term forecasting of network costs [28].

\section{CONCLUSIONS}

One of the main challenges of the literature about risk reduction in transport systems regards the development of ad-hoc TSMs able to simulate emergency conditions. In other words, models capable to capture the relevant changes of the network and of the users' behaviour, due to an approaching calamitous event.

The paper focuses on the supply (network) component of the TSMs. In relation to the consolidated static models, a modelling extension is necessary in order to capture two elements:

- the dynamics of the transportation network during the evacuation in terms of both topology (e.g. link closure, contraflow) and capacity of the links and of the network;

- the potentialities of emerging ICTs in providing valuable information according to both within-day and day-to-day patterns.

The concept of learning process has been introduced to take into account how the path costs (disutilities) in ordinary and emergency conditions will be perceived and updated by the users.

The new opportunities offered by the research on optimal path design in emergency conditions (e.g. during an evacuation) may be applied to incorporate inside the models the new potentialities offered by the emerging ICTs, such as the IoT to update within-day information, and the $\mathrm{BD}$ to update day-to-day information. 


\section{REFERENCES}

[1] Russo, F. \& Vitetta, A., Risk evaluation in a transportation system. International Journal of Sustainable Development and Planning, 1, pp. 170-191, 2006.

[2] Ortuzar, J. \& Willumsen, L.G., Modelling Transport, 3rd ed., Wiley: Chichester, 2001.

[3] Cascetta, E., Transportation Systems Analysis: Models and Applications, SpringerVerlag: New York, 2009.

[4] Cantarella, G.E., Watling, P.D., de Luca, S. \& Di Pace, R., Dynamics and Stochasticity in Transportation Systems: Tools for Transportation Network Modelling, Elsevier, 2020.

[5] Murray-Tuite, P. \& Mahmassani, H.S., Model of household trip-chain sequencing in emergency evacuation. Transportation Research Record, 1831(1), pp. 21-29, 2003.

[6] Kindinger, J.P., Programmatic Risk-Overview of Methods for Decision Making and Risk Management, Los Alamos National Laboratory document LA-CP-04-0626, 2004.

[7] Goldblatt, R., Evacuation planning: A key part of emergency planning. Proceedings of the Annual Meeting of Transportation Research Board, Washington, DC, pp. 1115, 2004.

[8] Lindell, M.K., Murray-Tuite, P., Wolshon, B. \& Baker, E.J., Large-Scale Evacuation: The Analysis, Modeling, and Management of Emergency Relocation from Hazardous Areas, Routledge, 2019.

[9] Russo, F. \& Rindone, C., The planning process and logical framework approach in road evacuation: A coherent vision. WIT Transactions on The Built Environment, vol. 117, WIT Press: Southampton and Boston, pp. 415-425, 2011.

[10] Russo, F. \& Rindone, C., Urban exposure: Training activities and risk reduction. WIT Transactions on Ecology and the Environment, vol. 191, WIT Press: Southampton and Boston, pp. 991-1001, 2014.

[11] Rindone, C. \& Panuccio, P., Risk reduction in transport system in emergency conditions: A framework for evacuation planning. WIT Transactions on the Built Environment, vol. 206, WIT Press: Southampton and Boston, submitted for publication.

[12] Russo, F. \& Rindone, C., Safety of users in road evacuation: The logical framework approach in evacuation planning. Proceedings of the 14th International Conference on Urban Transport and the Environment in the 21st Century, pp. 751-760, 2008.

[13] Russo, F. \& Rindone, C., Safety of users in road evacuation: Modelling and DSS for LFA in the planning process. WIT Transactions on Ecology and the Environment, vol. 120, WIT Press: Southampton and Boston, 2009.

[14] Russo, F. \& Chilà, G., Risk reduction in transport system in emergency conditions: A framework for demand analysis. WIT Transactions on the Built Environment, vol. 206, WIT Press: Southampton and Boston, submitted for publication.

[15] Marcianò, F.A., Musolino, G. \& Vitetta, A., A system of models for signal setting design of a signalized road network in evacuation conditions. WIT Transactions on the Built Environment, vol. 111, WIT Press: Southampton and Boston, pp. 313-323, 2010.

[16] Marcianò, F.A., Musolino, G. \& Vitetta, A., Within-day traffic assignment and signal setting in road evacuation: A procedure with explicit path enumeration. WIT Transactions on the Built Environment, vol. 117, WIT Press: Southampton and Boston, pp. 403-414, 2011.

[17] Di Gangi, M., Watling, D. \& Di Salvo, R., Modeling evacuation risk using a stochastic process formulation of mesoscopic dynamic network loading. IEEE Transactions on Intelligent Transportation Systems, 2020. 
[18] Di Gangi, M. \& Polimeni, A., A mesoscopic approach to model route choice in emergency conditions. Paper presented at the Springer Proceedings in Mathematics and Statistics, 217, pp. 547-555. 2017.

[19] Di Gangi, M. \& Belcore, O.M., Risk reduction in transport system in emergency conditions: A framework for decision support systems. WIT Transactions on the Built Environment, vol. 206, WIT Press: Southampton and Boston, submitted for publication.

[20] Musolino, G. \& Vitetta, A., Short-term forecasting in road evacuation: Calibration of a travel time function. WIT Transactions on the Built Environment, vol. 116, WIT Press: Southampton and Boston, pp. 615-626, 2011.

[21] Marcianò, F.A., Musolino, G. \& Vitetta A., Signal setting optimization on urban road transport networks: The case of emergency evacuation. Safety Science, 72, pp. 209220, 2015.

[22] Vitetta, A., Risk reduction in transport system in emergency conditions: A framework for network design problem. WIT Transactions on the Built Environment, vol. 206, WIT Press: Southampton and Boston, submitted for publication.

[23] Wardrop, J.G., Some theoretical aspects of road traffic research. Proc. Inst. Civ. Eng., 2(1), pp. 325-378, 1952.

[24] Cantarella, G.E. \& Cascetta, E., Dynamic processes and equilibrium in transportation networks. Transportation Science, 29, pp. 305-329, 1995.

[25] Russo, F. \& Comi, A., Providing dynamic route advice for urban goods vehicles: The learning process enhanced by the emerging technologies. Transportation Research Procedia - 24th EURO Working Group on Transportation Meeting, EWGT 2021, 8 10 September, Aveiro, Portugal, 2021.

[26] Russo, F. \& Musolino, G., The role of emerging ICT in the ports: Increasing utilities according to shared decisions. Front. Future Transp. 2, 722812, 2021 DOI: $10.3389 /$ ffutr.2021.722812.

[27] Carlan, V., Coppens, F., Sys, C., Vanelslander, T. \& Van Gastel, G., Blockchain technology as key contributor to the integration of maritime supply chain? Maritime Supply Chains, eds T. Vanelslander \& C. Sys, Elsevier, pp. 229-259, 2020.

[28] Atzori, L., Iera, A. \& Morabito, G., Understanding the Internet of Things: Definition, potentials, and societal role of a fast evolving paradigm. Ad Hoc Networks, 56, pp. 122-140, 2017. 